\title{
Alternating Copolymerization of Epoxides with Isothiocyanates
}

Chao Chen, Yves Gnanou, * and Xiaoshuang Feng*

Physical Sciences and Engineering Division, King Abdullah University of Science and Technology (KAUST), Thuwal 23955, Saudi Arabia

\section{Table of contents}

Table S1. Conditions of the isothiocyanate homopolymerization. 2

Figure S1. ${ }^{1} \mathrm{H}$ NMR spectra of (A) EITC, (B) HITC and (C) PITC reacted with P4 and $\mathrm{MBzOH}$ in $\mathrm{CDCl}_{3}$ .4

Figure S2. ${ }^{1} \mathrm{H}$ NMR spectrum of crude polymerization solution of Table 1 entry 1 in $\mathrm{CDCl}_{3}$ .4

Figure S3. ${ }^{1} \mathrm{H}$ NMR spectrum of PO-alt-EITC (Table 1, entry 1) in $\mathrm{CDCl}_{3} \ldots \ldots \ldots \ldots \ldots . . . .5$

Figure S4. ${ }^{1} \mathrm{H}$ NMR spectrum of PO-alt-HITC (Table 1, entry 2) in $\mathrm{CDCl}_{3} \ldots \ldots \ldots \ldots . . .5$

Figure S5. ${ }^{1} \mathrm{H}$ NMR spectra of PO-alt-PITC and polypropylene oxide in $\mathrm{CDCl}_{3} \ldots \ldots . .6$

Figure S6. FTIR spectrum of the PO-alt-PITC (Table 1, entry 6) ............................6

Figure S7. FTIR spectrum of the PO-alt-EITC (Table 1, entry 1) ............................

Figure S8. FTIR spectrum of the PO-alt-HITC (Table 1, entry 2) ...........................

Figure S9. ${ }^{1} \mathrm{H}-{ }^{13} \mathrm{C}$ HMBC spectrum of PO-alt-PITC (Table 1, entry 6). .............

Figure S10. ${ }^{1} \mathrm{H}$ NMR spectrum of CHO-alt-PITC (Table 1, entry 9) in $\mathrm{CDCl}_{3} \ldots \ldots \ldots . .8$

Figure S11. ${ }^{1} \mathrm{H}$ NMR spectrum of EO-alt-PITC (Table 1, entry 8) in $\mathrm{CDCl}_{3}$ .9 
Figure S12. FTIR spectrum of the CHO-alt-PITC (Table 1, entry 9) .........................

Figure S13. FTIR spectrum of the EO-alt-PITC (Table 1, entry 8) ..........................10

Figure S14. SEC traces of EO-alt-PITC (Table 1, entry 8) using DMF as the eluent and PS standards 10

Figure S15. SEC traces of poly(PO-alt-PITC) homopolymer (black) and poly(PO-altPITC)- $b$-(EO-alt-PITC) (red)

Figure S16. ${ }^{1} \mathrm{H}$ NMR spectrum of block copolymer poly(PO-alt-PITC)-b-(EO-altPITC) in $\mathrm{CDCl}_{3}$

Figure S17. Plots of $M_{\mathrm{n}}$ and $M_{\mathrm{w}} / M_{\mathrm{n}}$ vs. conversion of PITC into the copolymer in the presence of $\mathrm{ZnEt}_{2}$ 12

Figure S18. SEC traces of PO-alt-PITC copolymers with different molar masses in the presence of $\mathrm{ZnEt} 2$ 12 
Table S1. Conditions of the isothiocyanate homopolymerization. ${ }^{\mathrm{a}}$

\begin{tabular}{|c|c|c|c|c|c|}
\hline $\mathbf{L B}$ & Initiator & $\mathbf{T}\left({ }^{\circ} \mathbf{C}\right)$ & Isothiocyanate & I: ITC & Conversion $(\%)^{\mathrm{b}}$ \\
\hline P4 & MBzOH & 25 & EITC & $1: 100$ & 14 \\
\hline P4 & MBzOH & 25 & PITC & $1: 100$ & 0 \\
\hline P4 & MBzOH & 25 & HITC & $1: 100$ & 11 \\
\hline
\end{tabular}

a The homopolymerizations were run at $25{ }^{\circ} \mathrm{C}$ for $12 \mathrm{~h}$ unless otherwise noted. ${ }^{\mathrm{b}}$ Determined by ${ }^{1} \mathrm{H}$ NMR spectroscopy.

A<smiles>CCn1c(=S)n(CC)c(=S)n(CC)c1=S</smiles>

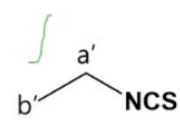

Cs

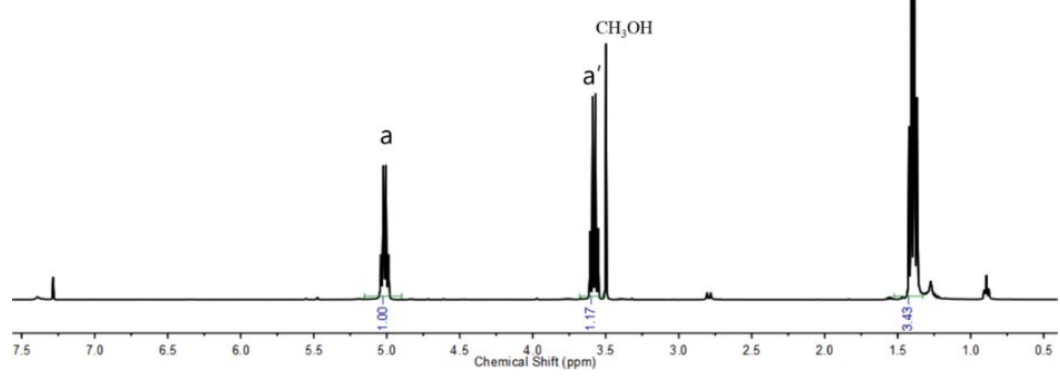

B

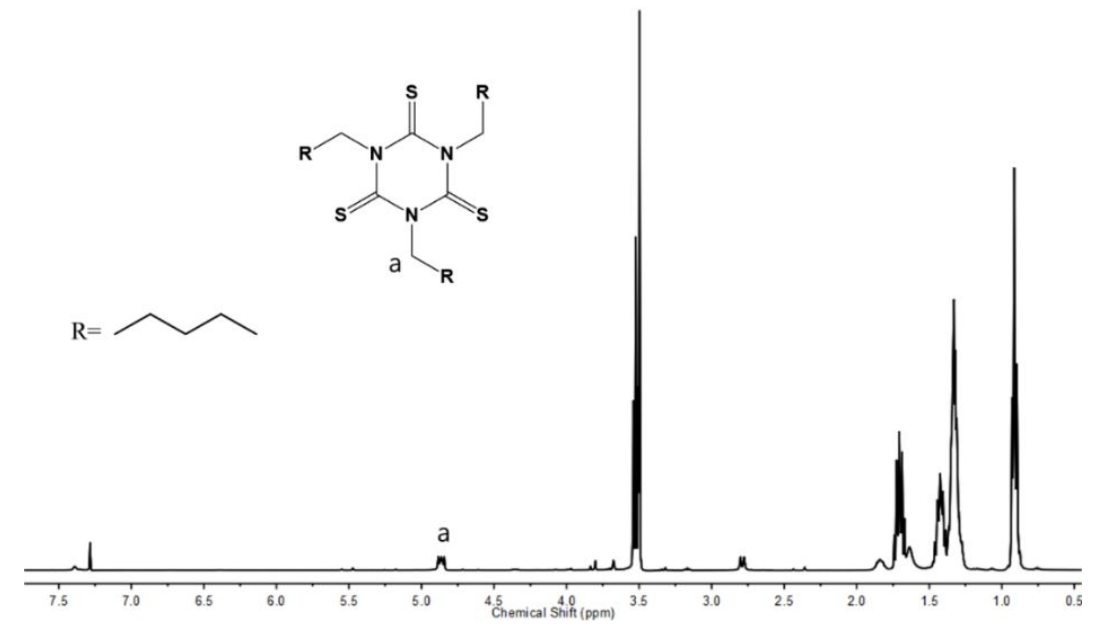




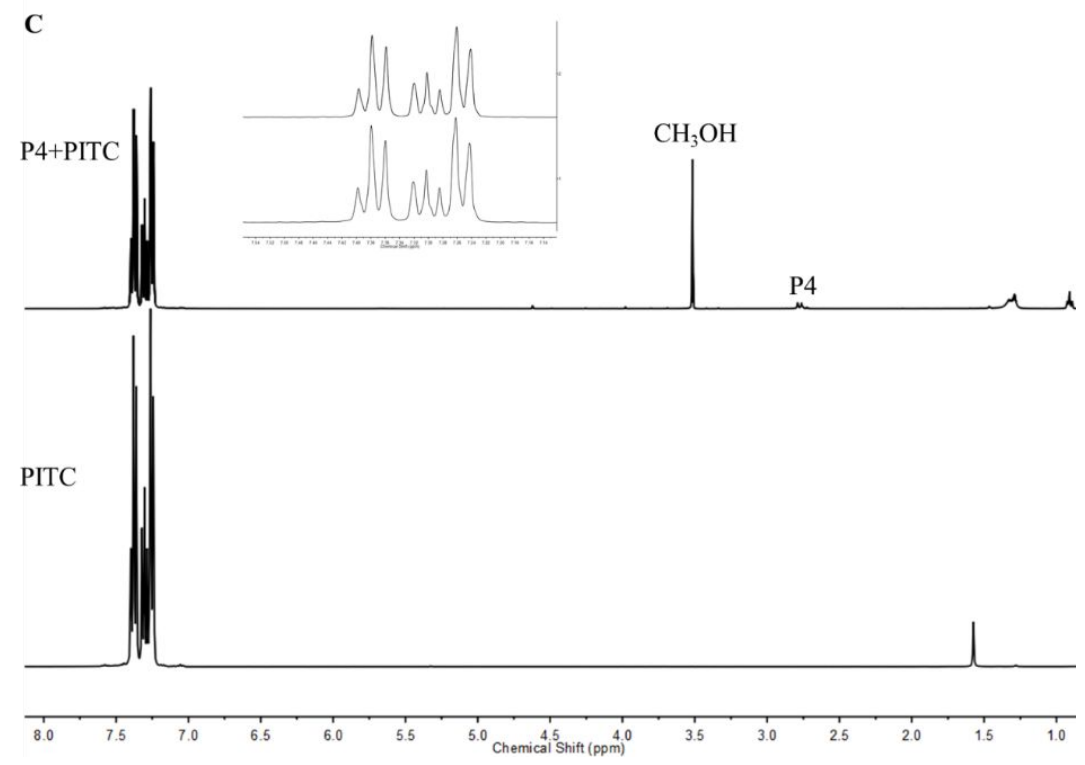

Figure S1. ${ }^{1} \mathrm{H}$ NMR spectra of (A) EITC, (B) HITC and (C) PITC reacted with P4 and $\mathrm{MBzOH}$ in $\mathrm{CDCl}_{3}$.<smiles>CCn1c(=S)n(CC)c(=S)n(CC)c1=S</smiles><smiles>CCN=C1OC(C)CP1</smiles>

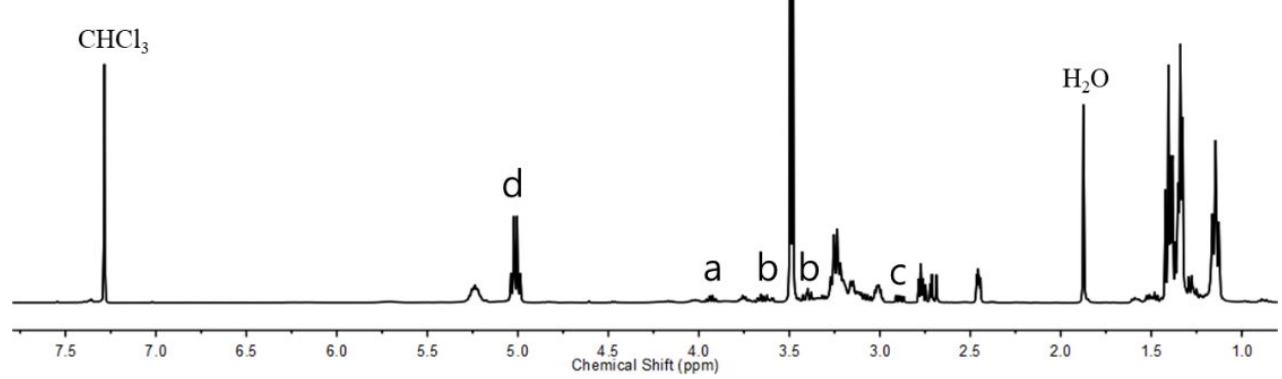

Figure S2. ${ }^{1} \mathrm{H}$ NMR spectrum of crude polymerization solution of Table 1 entry 1 in $\mathrm{CDCl}_{3}$. 

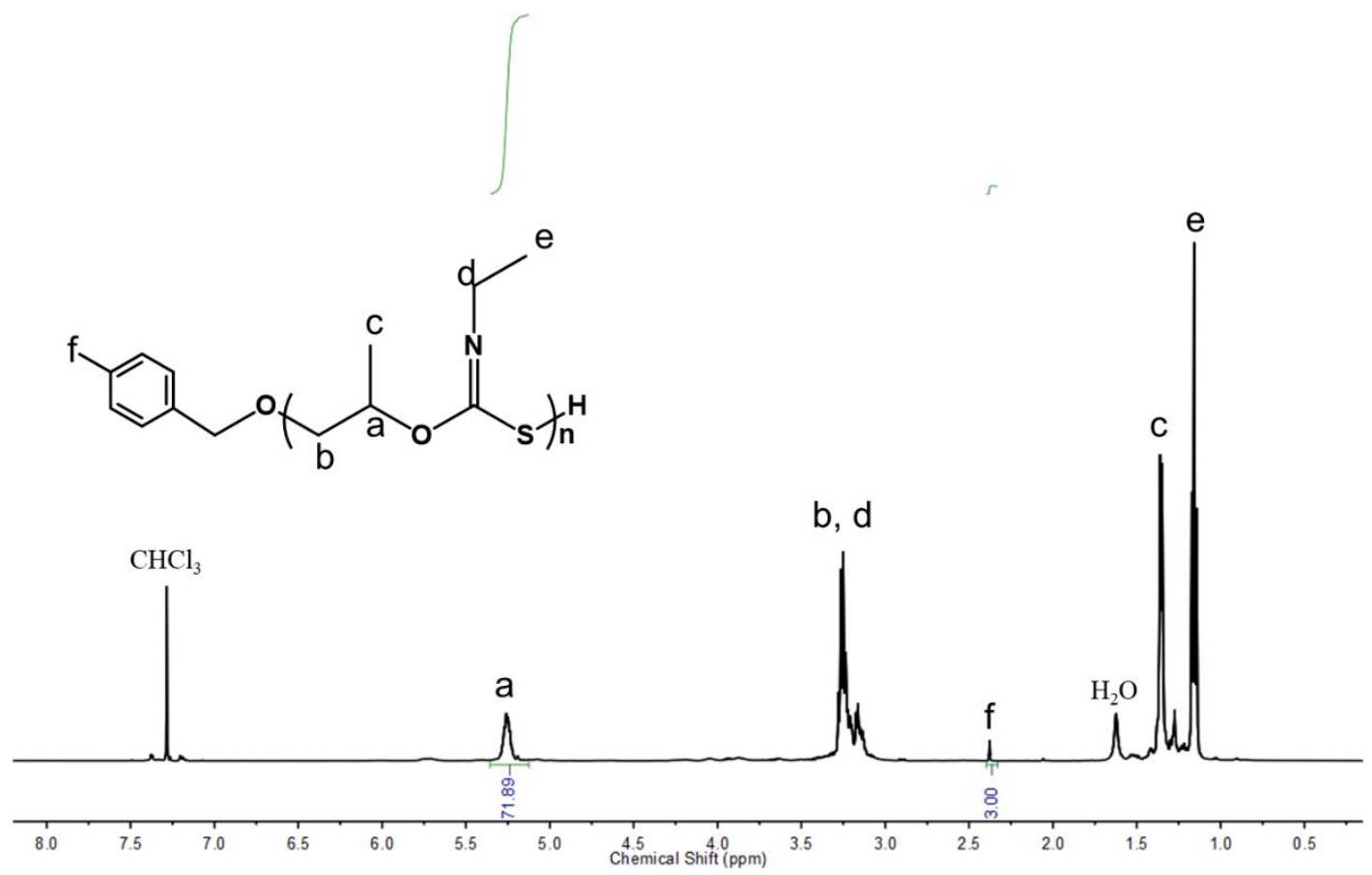

Figure S3. ${ }^{1} \mathrm{H}$ NMR spectrum of PO-alt-EITC (Table 1, entry 1) in $\mathrm{CDCl}_{3}$.

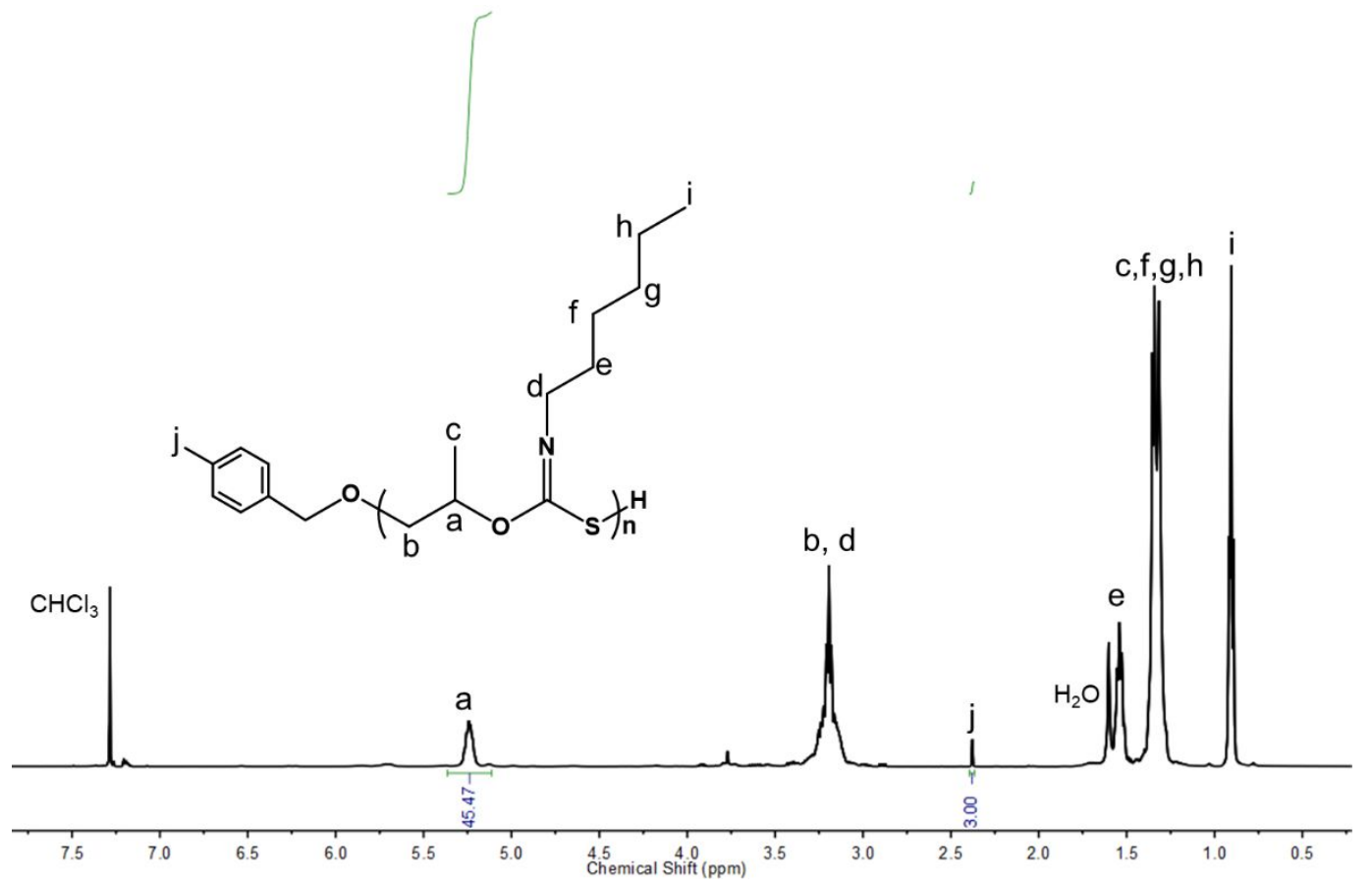

Figure S4. ${ }^{1} \mathrm{H}$ NMR spectrum of PO-alt-HITC (Table 1, entry 2) in $\mathrm{CDCl}_{3}$. 


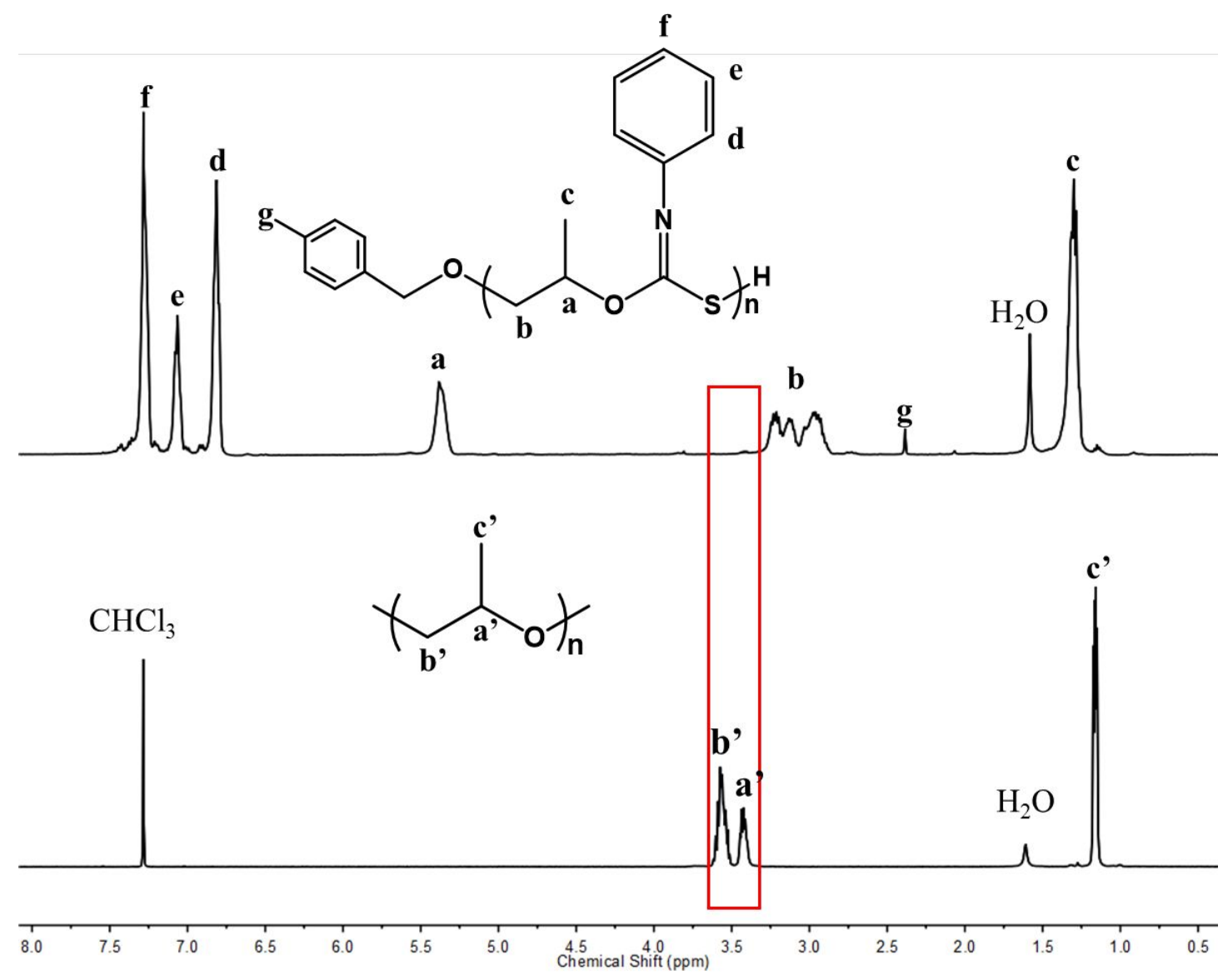

Figure S5. ${ }^{1} \mathrm{H}$ NMR spectra of PO-alt-PITC and polypropylene oxide in $\mathrm{CDCl}_{3}$.

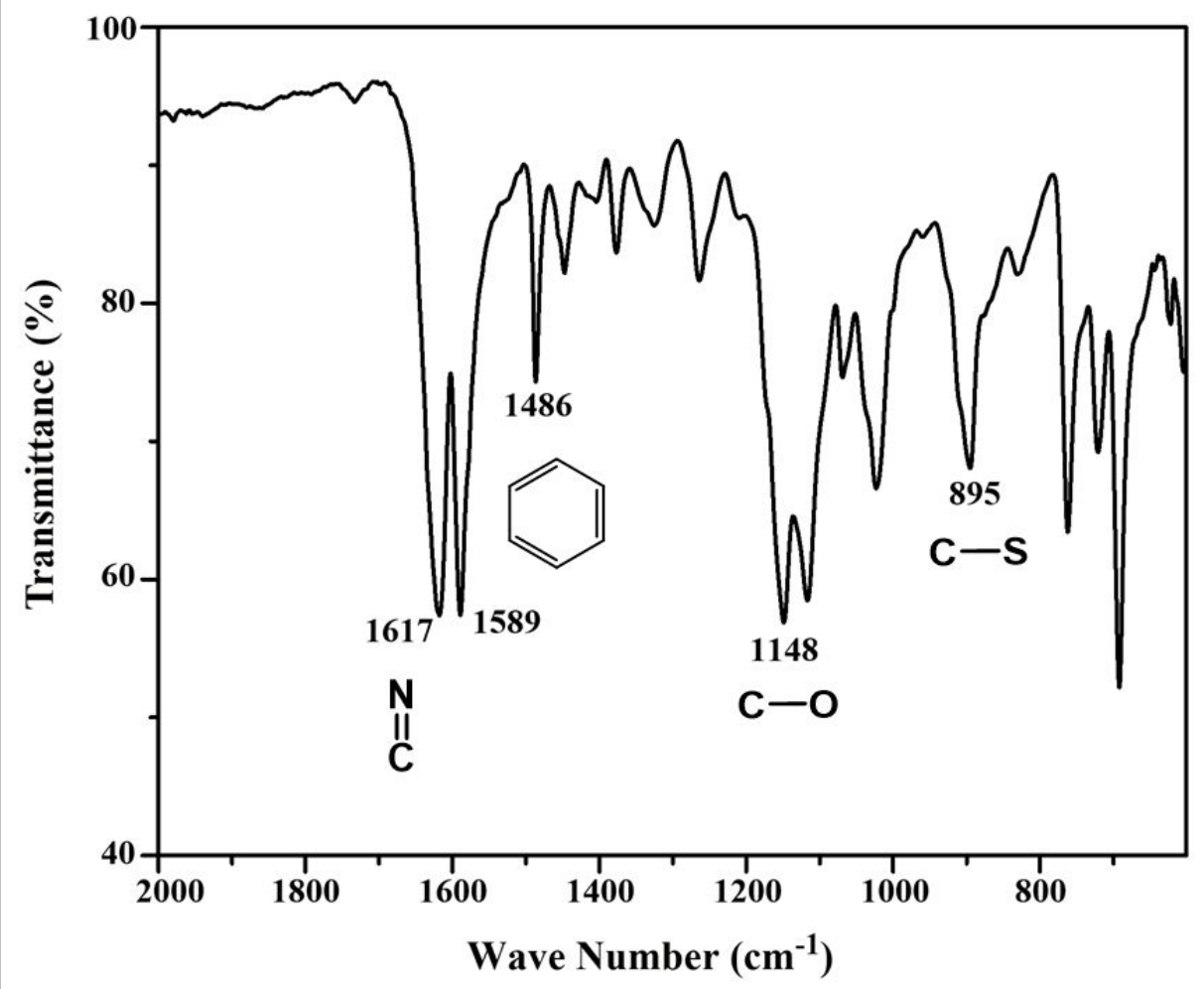

Figure S6. FTIR spectrum of the PO-alt-PITC (Table 1, entry 6). 


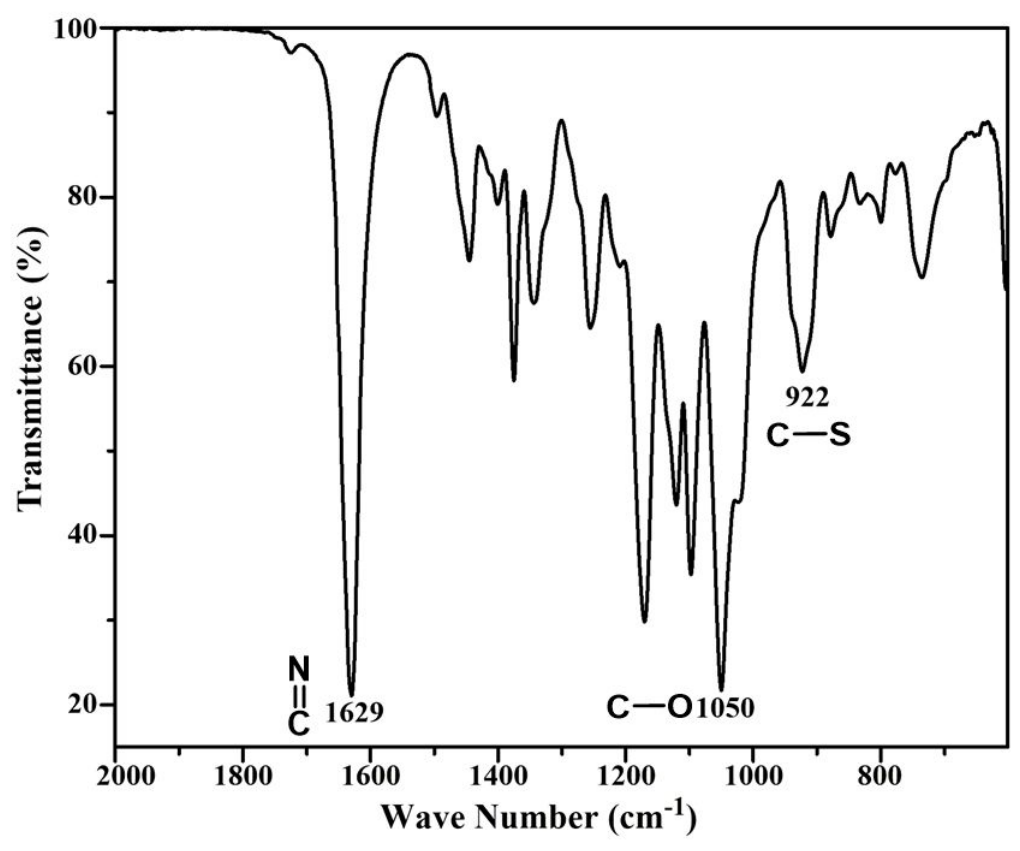

Figure S7. FTIR spectrum of the PO-alt-EITC (Table 1, entry 1).

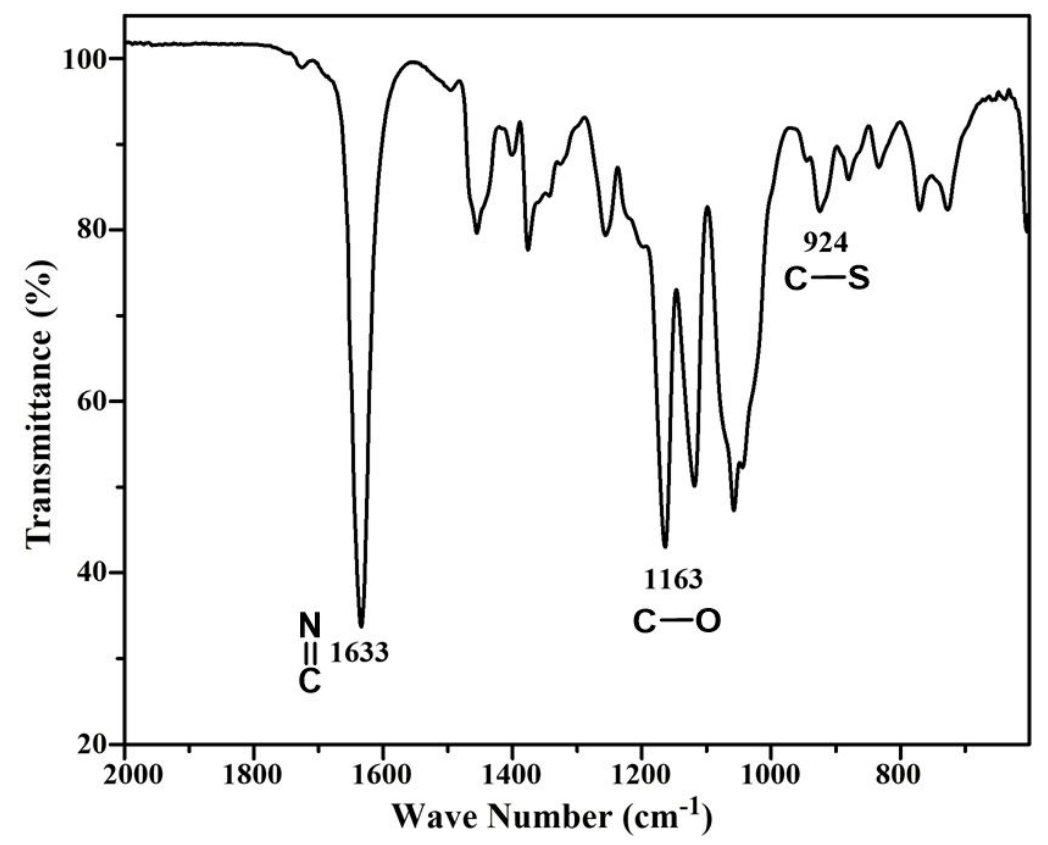

Figure S8. FTIR spectrum of the PO-alt-HITC (Table 1, entry 2). 


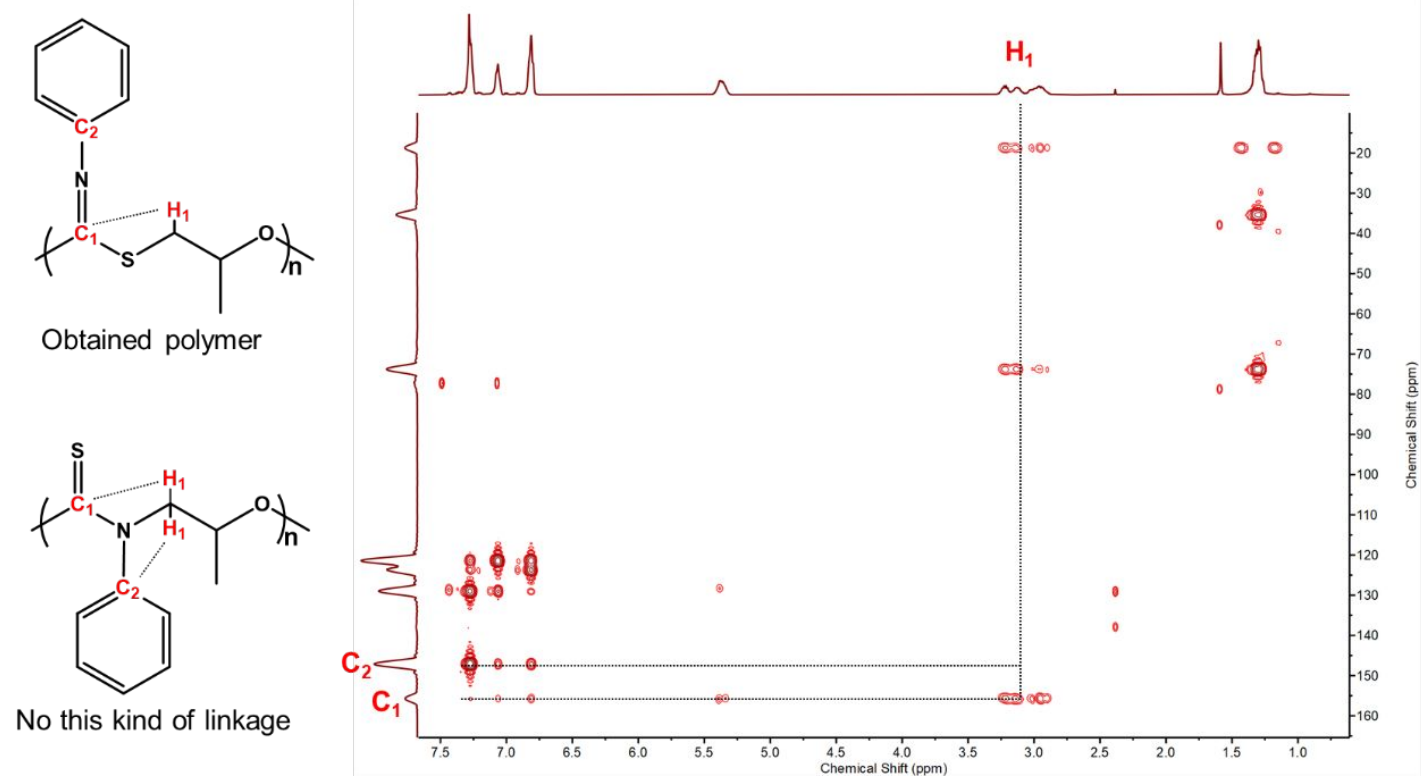

Figure S9. ${ }^{1} \mathrm{H}-{ }^{13} \mathrm{C}$ HMBC spectrum of PO-alt-PITC (Table 1, entry 6). No signal cross of the black dash lines reveal that no correlation between resonances of $\mathrm{H}_{1}$ and $\mathrm{C}_{2}$.

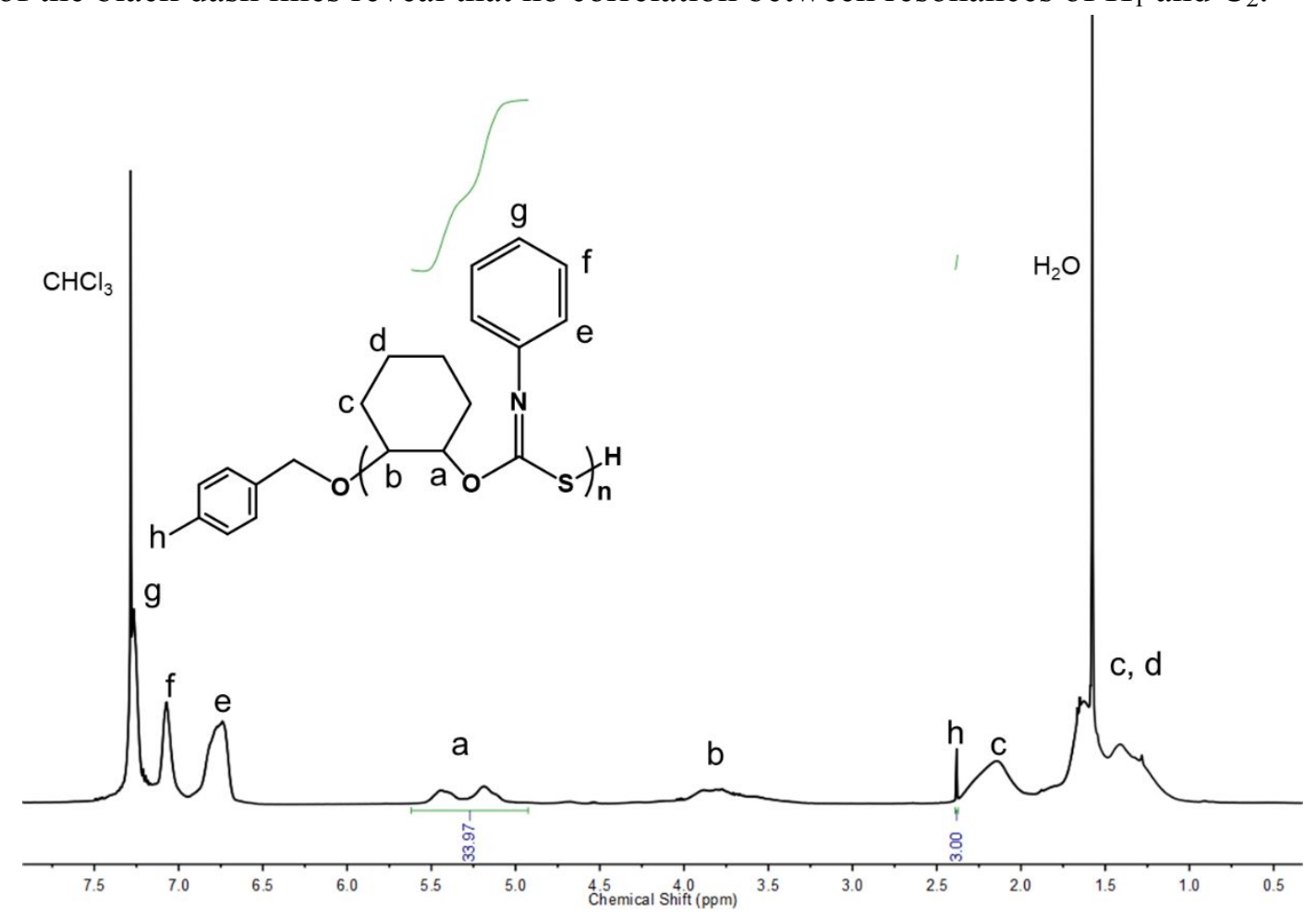

Figure S10. ${ }^{1} \mathrm{H}$ NMR spectrum of CHO-alt-PITC (Table 1, entry 9) in $\mathrm{CDCl}_{3}$. 


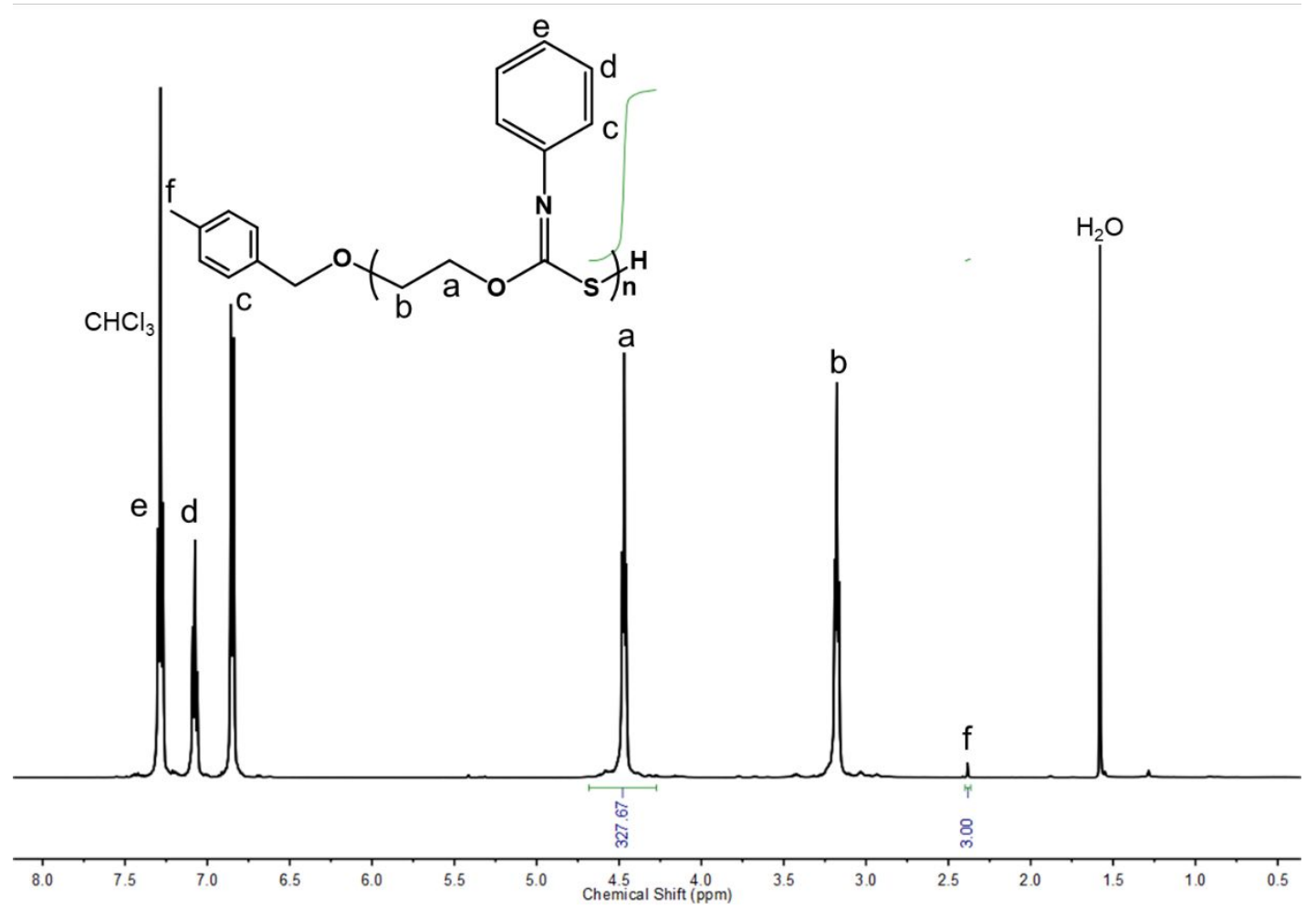

Figure S11. ${ }^{1} \mathrm{H}$ NMR spectrum of EO-alt-PITC (Table 1, entry 8) in $\mathrm{CDCl}_{3}$.

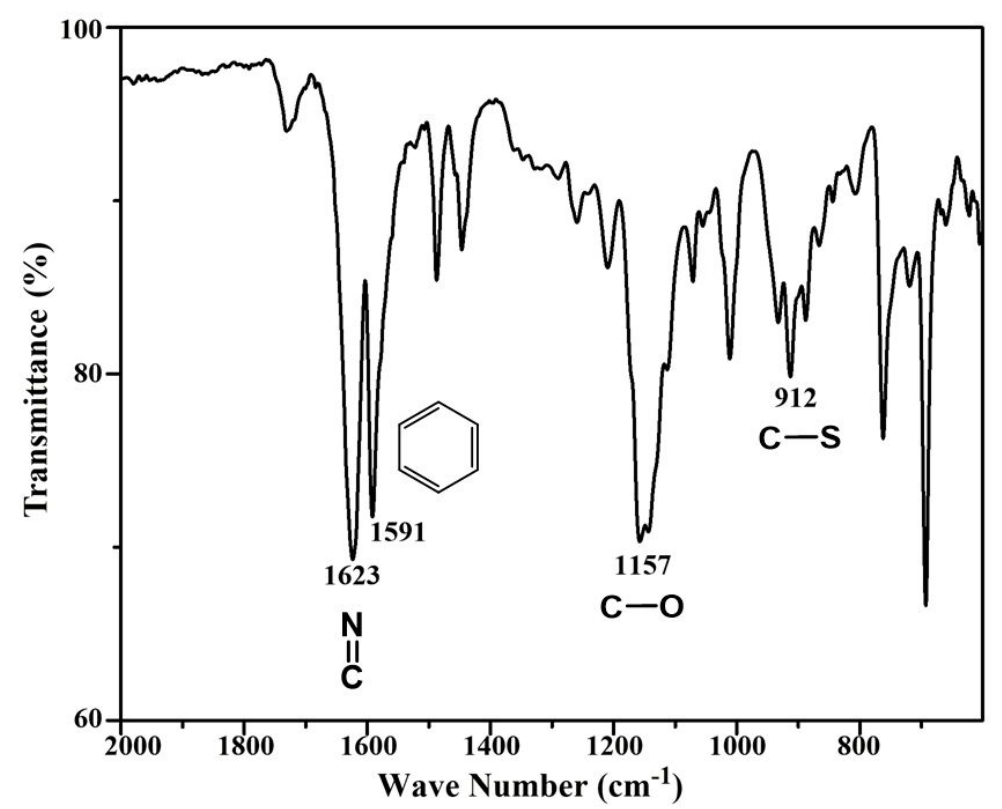

Figure S12. FTIR spectrum of the CHO-alt-PITC (Table 1, entry 9). 


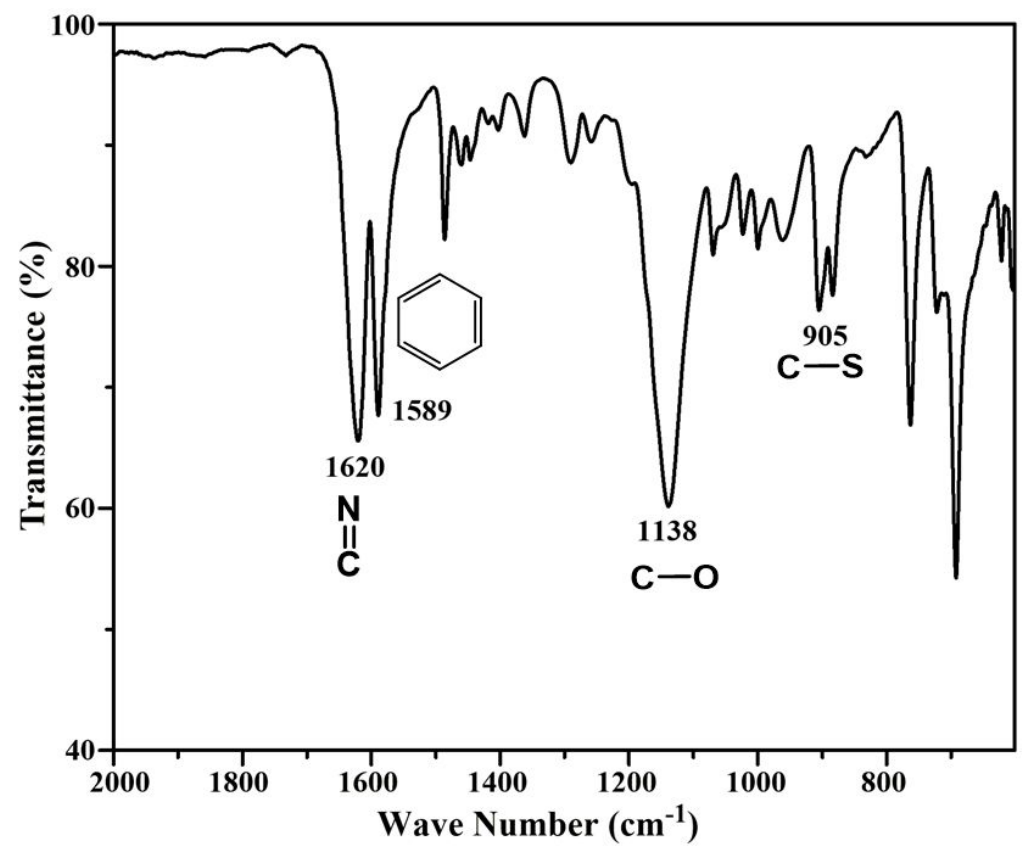

Figure S13. FTIR spectrum of the EO-alt-PITC (Table 1, entry 8).

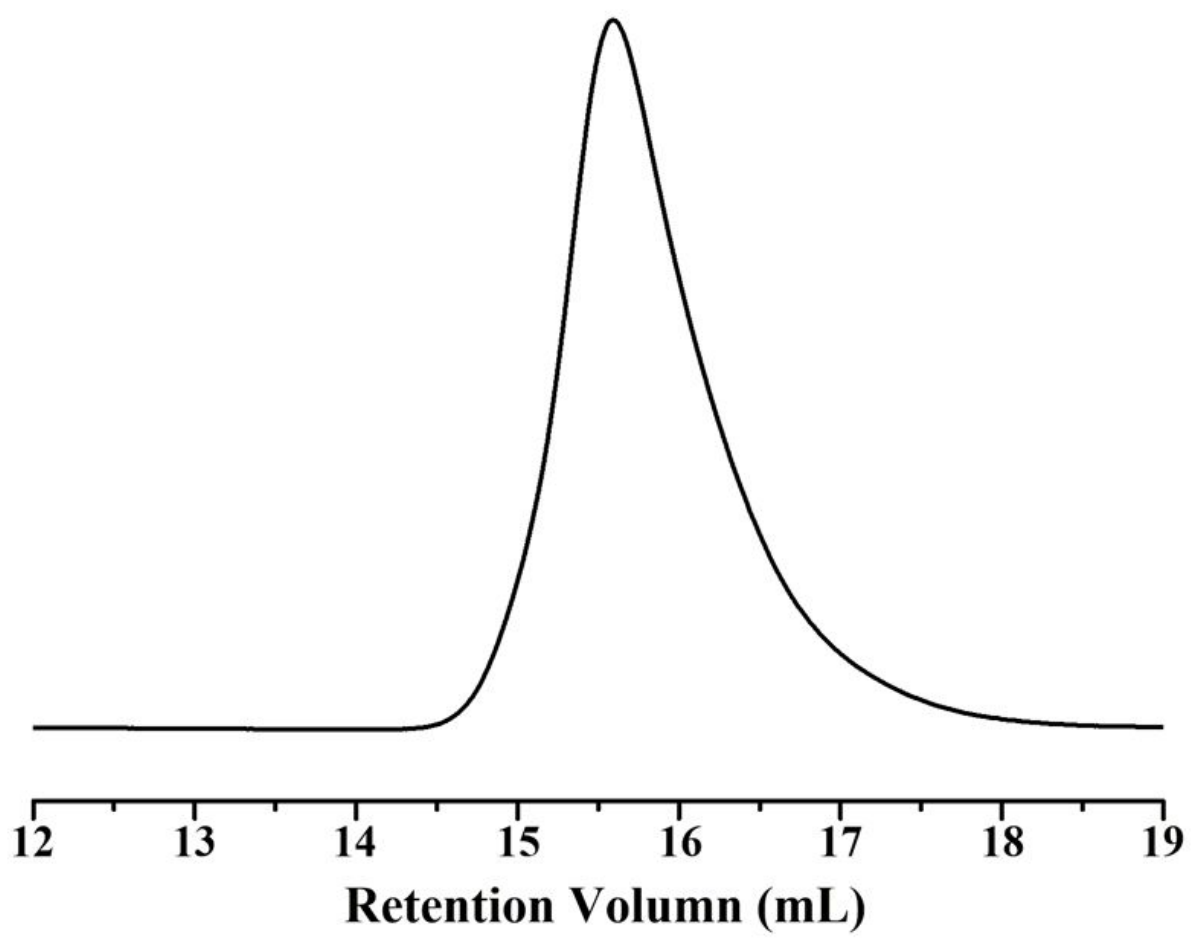

Figure S14. SEC traces of EO-alt-PITC (Table 1, entry 8) using DMF as the eluent and PS standards. 


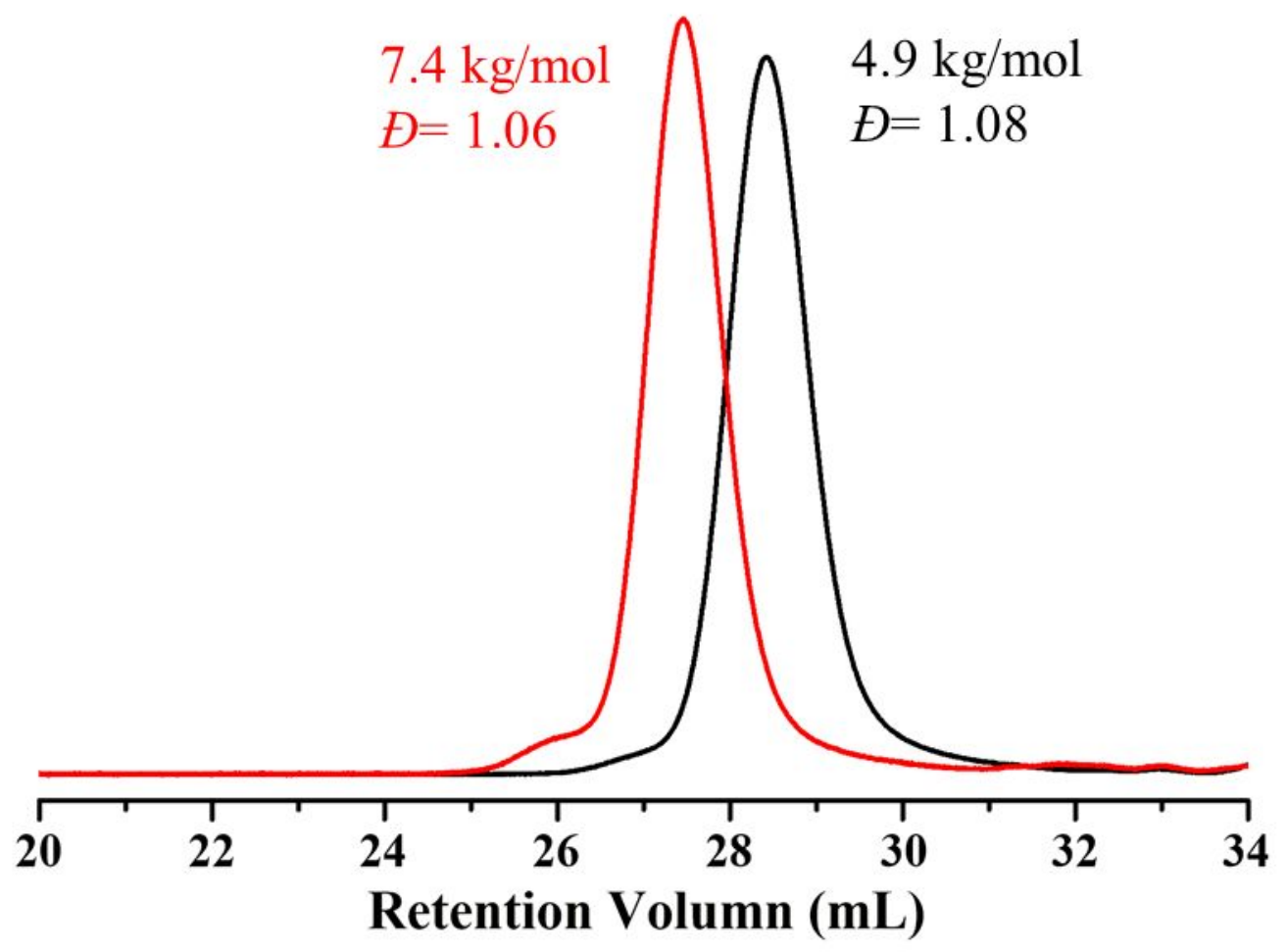

Figure S15. SEC traces of poly(PO-alt-PITC) homopolymer (black) and poly(PO-altPITC)- $b$-(EO-alt-PITC) (red).

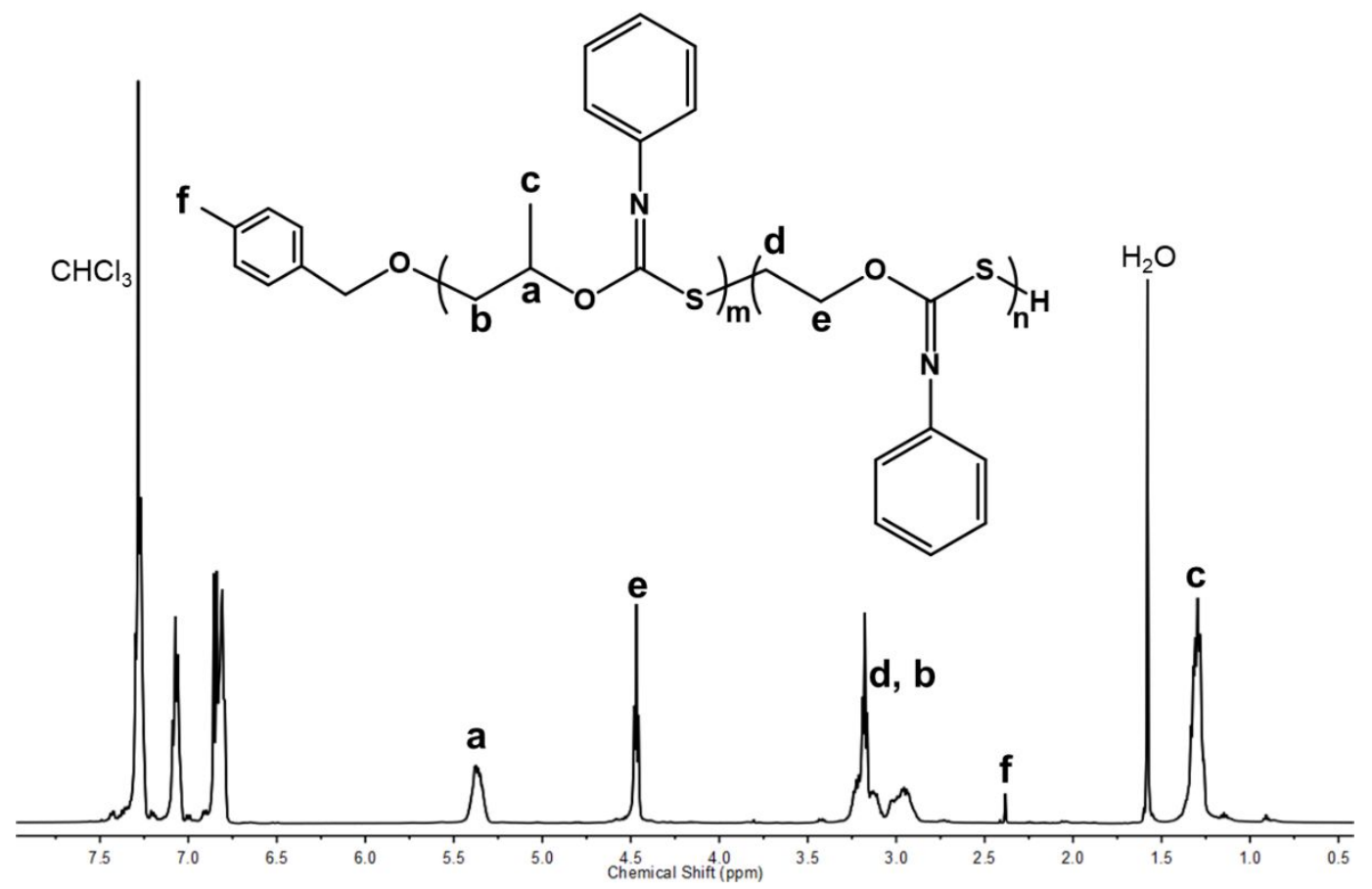

Figure S16. ${ }^{1} \mathrm{H}$ NMR spectrum of block copolymer poly(PO-alt-PITC)-b-(EO-altPITC) in $\mathrm{CDCl}_{3}$. 


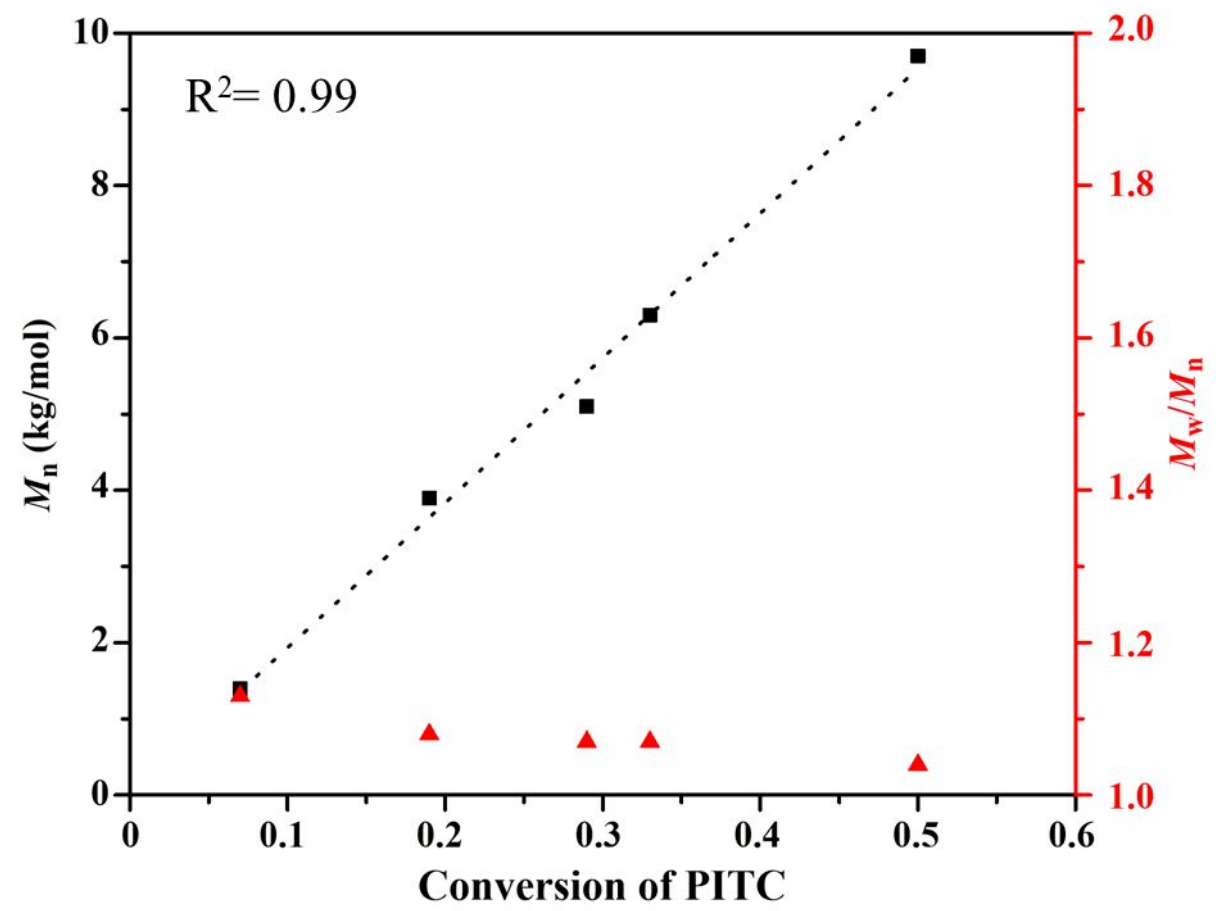

Figure S17. Plots of $M_{\mathrm{n}}$ and $M_{\mathrm{w}} / M_{\mathrm{n}}$ vs. conversion of PITC into the copolymer in the presence of $\mathrm{ZnEt} 2$.

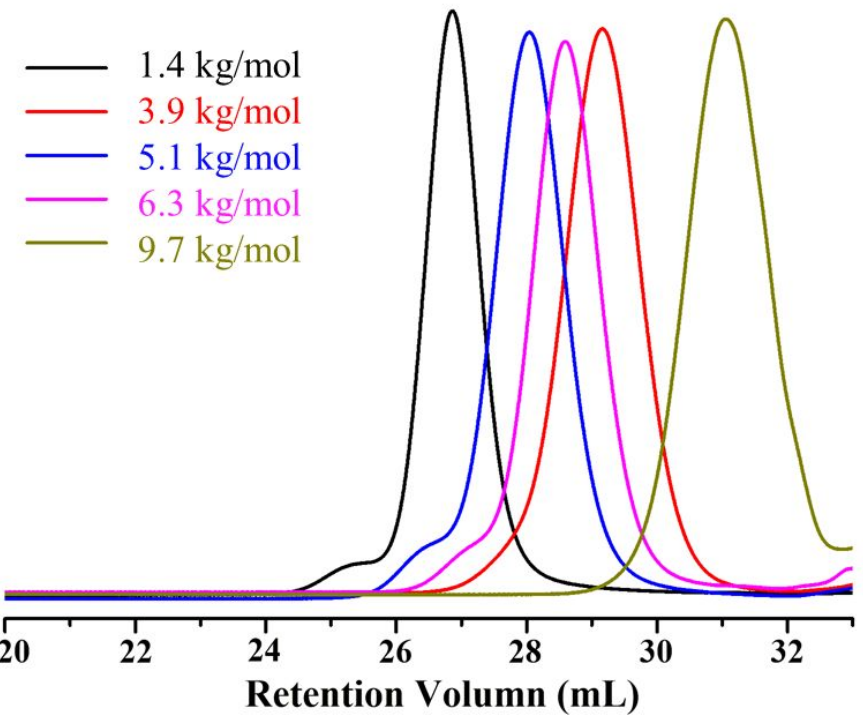

Figure S18. SEC traces of PO-alt-PITC copolymers with different molar masses in the presence of ZnEt2. 